\title{
Complicações do cateterismo venoso central em usuários de nutrição parenteral
}

\author{
Complications of central venous catheterization in users of parenteral nutrition
}

Complicaciones del cateterismo venoso central en usuarias de nutrición parenteral

\begin{abstract}
Auriane de Sousa Alencar ${ }^{1 *}$, Luis Gustavo Cavalcante Reinaldo ${ }^{1}$, Julciele Cícera da Silva ${ }^{1}$, Carlos Henrique Ferreira ${ }^{1}$.
\end{abstract}

\section{RESUMO}

Objetivo: Avaliar as complicações do cateterismo venoso central em usuários de nutrição parenteral. Métodos: Delineamento transversal analítico, com coleta de dados retrospectivos em fevereiro de 2021. A população do estudo compreendeu pacientes internados que utilizaram nutrição parenteral de janeiro de 2018 a dezembro de 2019. Uma amostra de conveniência composta por 70 participantes foi avaliada. Foram coletadas variáveis demográficas, clínicas, relacionadas à nutrição parenteral e investigação de complicações. Foram calculadas estatísticas uni e bivariadas. Resultados: A prevalência de complicações relacionadas ao cateter venoso central utilizado para nutrição parenteral foi de $51,4 \%$, sendo todas infecciosas. Predominou o sexo masculino (72,2\% e $76,5 \%)$ e diagnósticos de neoplasias gástrica $(27,8 \%$ e $29,4 \%$ ) e de pâncreas (13,9\% e 14,7\%) nos grupos com e sem complicações respectivamente. Número de sítios de inserção utilizados $(p=0,041)$, cateter em jugular esquerda $(p=0,014)$ e realização de troca de cateter $(p=0,024)$ foram relacionados à ocorrência de complicações. A espécie mais frequente foi Staphylococcus epidermidis (15,7\%). Conclusão: A prevalência de complicações dos cateteres de acesso venoso central para uso de nutrição parenteral foi alta e do tipo infecciosa. Maior número de sítios de inserção utilizados, acesso em jugular esquerda e realização de troca de cateter foram relacionados à ocorrência de complicações.

Palavras-chave: Nutrição parenteral, Cateterismo venoso central, Staphylococcus epidermidis.

\begin{abstract}
Objective: To evaluate the complications of central venous catheterization in patients using parenteral nutrition. Methods: Analytical cross-sectional design, with retrospective data collection in February 2021. The study population comprised patients who used parenteral nutrition in January 2018 to December 2019. A convenience sample of 70 participants was evaluated. Demographic and clinical variables, data on parenteral nutrition and investigation of complications were collected. Univariate and bivariate statistics were calculated. Results: The prevalence of complications related to the central venous catheter used for parenteral nutrition was $51.4 \%$ and all were infectious. Male $(72.2 \%$ and $76.5 \%)$ and diagnoses of gastric $(27.8 \% 29.4 \%)$ and pancreas $(13.9 \%$ and $14.7 \%)$ neoplasms predominated in groups with and without complications. Number of insertion sites $(p=0.041)$, catheter in the left jugular $(p=0.014)$ and catheter exchange $(p=0.024)$ were related to the occurrence of complications. The most frequent species was Staphylococcus epidermidis (15.7\%). Conclusion: The prevalence of complications of central venous access catheters for the use of parenteral nutrition was high and of the infectious type. A greater number of insertion sites used, access to the left jugular and catheter exchange were related to the occurrence of complications.
\end{abstract}

Keywords: Parenteral nutrition, Catheterization central venous, Staphylococcus epidermidis.

${ }^{1}$ Hospital Universitário da Universidade Federal do Piauí, Teresina - PI. *E-mail: aurialencar@hotmail.com 


\section{RESUMEN}

Objetivo: Evaluar las complicaciones del cateterismo venoso central en usuarios de nutrición parenteral. Métodos: Diseño analítico de corte transversal, con recolección de datos retrospectiva en febrero de 2021. La población de estudio estuvo conformada por pacientes hospitalizados que utilizaron nutrición parenteral desde enero de 2018 a diciembre de 2019. Se evaluó una muestra de conveniencia de 70 participantes. Se recogieron variables demográficas y clínicas relacionadas con la nutrición parenteral y la investigación de complicaciones. Se calcularon estadísticas univariadas y bivariadas. Resultados: La prevalencia de complicaciones relacionadas con el catéter venoso central utilizado para la nutrición parenteral fue del $51,4 \%$ y todas fueron infecciosas. Las neoplasias masculinas $(72,2 \%$ y $76,5 \%)$ y los diagnósticos de neoplasias gástricas $(27,8 \% 29,4 \%)$ y páncreas $(13,9 \%$ y $14,7 \%)$ predominaron en los grupos con y sin complicaciones. Número de sitios de inserción ( $p=0,041)$, catéter en la yugular izquierda $(p=0,014)$ e intercambio de catéter $(p=0,024)$ se relacionaron con la aparición de complicaciones. La especie más frecuente fue Staphylococcus epidermidis (15,7\%). Conclusión: La prevalencia de complicaciones de los catéteres de acceso venoso central para el uso de nutrición parenteral fue alta y de tipo infeccioso. Un mayor número de sitios de inserción utilizados, el acceso a la yugular izquierda y el reemplazo del catéter se relacionaron con la aparición de complicaciones.

Palabras clave: Nutrición parenteral, Cateterismo venoso central, Staphylococcus epidermidis.

\section{INTRODUÇÃO}

A nutrição parenteral é uma forma de alimentação na qual a estimativa das necessidades calóricas totais ou parciais é alcançada por meio da administração intravenosa de nutrientes, indicada para pacientes que apresentam impossibilidade de utilização do trato gastrointestinal. O que pode estar aliado a outras situações clínicas, como desnutrição com perda massa corpórea acima de $15 \%$ e estados hipercatabólicos. Por se tratar de um tipo de nutrição que necessita da via intravenosa, a inserção de cateter vascular preferencialmente central é necessária para viabilizar a oferta dos nutrientes (ITZHAKI M e SINGER P, 2020; WEBER TK, et al., 2020). Contudo, é crescente a preocupação global quanto ao risco aumentado de complicações associadas ao cateter venoso central em pacientes que utilizam nutrição parenteral, em especial, no ambiente hospitalar (PATEL AR, et al., 2019).

Os cateteres venosos centrais são dispositivos muito utilizados em ambientes hospitalares que permitem o acesso à corrente sanguínea para a administração de medicamentos, fluidoterapia, nutrição parenteral, monitoração hemodinâmica ou hemodiálise. Esses dispositivos podem ser classificados quanto ao tipo de implantação em semi-implantados e totalmente implantados; quanto ao tempo de duração, em cateteres de curta duração (permanência de 15 dias) ou cateteres de longa duração (permanência além de 30 dias); e quanto ao número de lúmens, que pode ser de um a quatro (ZERATI AE, et al., 2017; MIYAO H, 2016).

As emulsões para nutrição parenteral oferecem habitualmente osmolaridades que necessitam de vasos calibrosos para sua infusão. Preferencialmente, utiliza-se acesso de veia subclávia, pois permite maior conforto à mobilização do paciente e um menor índice de infecção. De modo geral, o lúmen do cateter utilizado para nutrição parenteral é exclusivo para seu uso devido ao risco de incompatibilidades físico-químicas, como a formação de precipitados com a administração concomitante de medicações (LAMBELL KJ, et al., 2020).

Nesse contexto, apesar dos benefícios da utilização dos cateteres vasculares, não há como descartar os riscos. Diversos são os fatores de risco relacionados às complicações do cateter venoso central, como a escolha da veia puncionada, o tempo prolongado de uso, número de lúmens, tipo de fluido, doença de base do paciente e comorbidades. Estudos mostram que a inserção desses cateteres está relacionada a complicações que podem ser mecânicas, trombóticas e infecciosas (COMERLATO PH, et al., 2017). A complicação infecciosa destaca-se pelo impacto na morbimortalidade e nos custos da assistência à saúde, com prolongamento da hospitalização. Os cateteres de infusão de nutrição parenteral apresentam, ainda, um risco adicional por serem fontes de nutrientes que podem contribuir para o crescimento microbiano (COMERLATO PH, et al., 2020). 
A manutenção do cateter venoso central para infusão de nutrição parenteral tem sido uma complicação frequentemente observada na literatura, em diferentes contextos (SOU V, et al., 2017; PIRONI L, et al., 2020). Ademais, há uma importante taxa de mortalidade associada às complicações do cateterismo venoso central em usuários de nutrição parenteral (LAMBELL KJ, et al., 2020).

Nesse sentido, é pertinente somar evidências à literatura preexistente quanto aos potenciais danos provenientes de complicações relacionadas ao cateterismo venoso central nos pacientes usuários de nutrição parenteral, na perspectiva de direcionar esforços à estruturação de protocolos para a melhoria da qualidade assistencial, com atenção especial à segurança do paciente. Assim, este estudo objetivou avaliar as complicações do cateterismo venoso central em usuários de nutrição parenteral internados em um Hospital Universitário do Nordeste do Brasil.

\section{MÉTODOS}

Delineamento transversal analítico, com coleta de dados retrospectivos, realizado na Unidade de Nutrição Clínica em janeiro de 2021, e avaliou pacientes que utilizaram nutrição parenteral por cateter venoso central no período de janeiro de 2018 a dezembro de 2019, correspondendo a 70 pacientes.

Foram critérios de inclusão da amostra: ter idade igual ou superior a 18 anos e ter utilizado nutrição parenteral por cateter venoso central. Foi critério de exclusão apresentar dados ausentes em prontuário referentes à nutrição parenteral que prejudicassem as análises.

Foi utilizado um formulário de coleta de dados, contendo variáveis demográficas (idade e sexo), clínicas (diagnóstico e estado nutricional) e relacionadas à nutrição parenteral por cateter venoso central (indicação da nutrição parenteral, diárias de nutrição parenteral, sítios de inserção utilizados, troca de cateter, complicação relacionada ao cateter e desfecho clínico). Além disso, foram verificados dados de investigação da complicação (preenchimento do formulário de atividades preventivas padronizadas, ponta de cateter, hemocultura, linfócitos, albumina e microrganismo). A avaliação nutricional contemplou o cálculo do Índice de massa corporal (IMC).

A coleta de dados foi realizada em fontes de informações intra-hospitalares e base de dados própria da Equipe Multidisciplinar de Terapia Nutricional para identificação dos pacientes que atendiam aos critérios do estudo.

Os dados foram processados utilizando o software IBM ${ }^{\circledR}$ SPSS $\AA$, versão 26.0. Foram calculadas estatísticas univaridas, como média, desvio padrão, mínimo e máximo, para as variáveis quantitativas, e frequências para as qualitativas. $\mathrm{Na}$ análise inferencial, a variável dependente foi definida como a "presença de complicação". Para as variáveis independentes quantitativas, foi utilizado o teste de Kolmogorov-Smirnov para verificação da normalidade dos dados e foi realizado o teste $t$ de Student. Para as variáveis independentes qualitativas, foi realizado o teste Qui-Quadrado de Parson e, quando não atendidos os pressupostos deste teste, foi realizado o teste Exato de Fisher. Para as associações significativas, foi calculada a Razão de Prevalência (RP) e seu respectivo intervalo de confiança (IC95\%). Foram consideradas significativas as relações com $p<0,05$.

Foram seguidas as normatizações éticas nacionais e internacionais para pesquisas com seres humanos. O estudo foi autorizado pelo hospital universitário e foi aprovado por Comitê de Ética em Pesquisa, sob parecer $n .-4525360 / 2021$. Todos os participantes assinaram o termo de consentimento livre e esclarecido, em duas vias, e foi apresentado o termo de compromisso de utilização de dados.

\section{RESULTADOS}

Complicações relacionadas ao cateter venoso central utilizado para nutrição parenteral ocorreram em 36 $(51,4 \%)$ pacientes internados no hospital universitário, em que $35(50,0 \%)$ foram somente infecciosas e 1 $(1,4 \%)$ compreendeu uma complicação infecciosa concomitantemente a uma intolerância hepática. Na Tabela 1, apresenta as características demográficas e clínicas dos pacientes. 
Tabela 1 - Variáveis demográficas e clínicas dos pacientes internados usuários de nutrição parenteral por cateter venoso central conforme presença de complicação $(n=70)$.

\begin{tabular}{|c|c|c|c|}
\hline Variável & $\begin{array}{l}\text { Com complicação } \\
(n=36)\end{array}$ & $\begin{array}{c}\text { Sem complicação } \\
(n=34)\end{array}$ & $p$ \\
\hline Idade $(\mathrm{M} \pm \mathrm{DP})$ & $54,1 \pm 17,6$ & $58,7 \pm 14,3$ & $0,242^{t}$ \\
\hline Sexo & & & $0,684^{9}$ \\
\hline Masculino & $26(72,2 \%)$ & $26(76,5 \%)$ & \\
\hline Feminino & $10(27,8 \%)$ & $8(23,5 \%)$ & \\
\hline Diagnóstico & & & $0,648^{q}$ \\
\hline Neoplasia gástrica & $10(27,8 \%)$ & $10(29,4 \%)$ & \\
\hline Neoplasia de pâncreas & $5(13,9 \%)$ & $5(14,7 \%)$ & \\
\hline Neoplasia de peritoneal & - & $2(5,9 \%)$ & \\
\hline Neoplasia de esôfago & $1(2,8 \%)$ & - & \\
\hline Neoplasia de cólon & $1(2,8 \%)$ & $2(5,9 \%)$ & \\
\hline Neoplasia de ovário & $1(2,8 \%)$ & $1(2,9 \%)$ & \\
\hline Neoplasia óssea & $1(2,8 \%)$ & - & \\
\hline Neoplasia de reto & $1(2,8 \%)$ & - & \\
\hline Neoplasia de laringe & - & $1(2,9 \%)$ & \\
\hline Neoplasia de intestino delgado & - & $1(2,9 \%)$ & \\
\hline Fístula enterocutânea & $2(5,6 \%)$ & $1(2,9 \%)$ & \\
\hline Coledocolitiase & $1(2,8 \%)$ & $3(8,8 \%)$ & \\
\hline Pancreatite & $2(5,6 \%)$ & $1(2,9 \%)$ & \\
\hline Síndrome do intestino curto & $1(2,8 \%)$ & - & \\
\hline Doença de Crohn & $5(13,9 \%)$ & $1(2,9 \%)$ & \\
\hline Celulite orbitrária & $1(2,8 \%)$ & - & \\
\hline Nódulo hepático benigno & & $1(2,9 \%)$ & \\
\hline Colostomia/lleostomia & $1(2,8 \%)$ & $2(5,9 \%)$ & \\
\hline Doença de Chagas & $1(2,8 \%)$ & - & \\
\hline Fístula mucosa & - & $1(2,9 \%)$ & \\
\hline Cirrose & $1(2,8 \%)$ & - & \\
\hline Acalasia & - & $1(2,9 \%)$ & \\
\hline Estenose duodenal/esofágica & $1(2,8 \%)$ & $1(2,9 \%)$ & \\
\hline Estado nutricional & & & $0,130^{q}$ \\
\hline Baixo peso & $16(44,4 \%)$ & $22(64,7 \%)$ & \\
\hline Eutrofia & $19(52,8 \%)$ & $10(29,4 \%)$ & \\
\hline Excesso de peso/obeso & $1(2,8 \%)$ & $2(5,9 \%)$ & \\
\hline
\end{tabular}

Legenda: M $\pm D P$ : média \pm desvio padrão; $p$ : significância do teste; t: teste t de Student; q: Qui-Quadrado de Pearson; -: distribuição de frequências não atendeu aos requisitos dos testes.

Fonte: Alencar AS, et al., 2021.

Os pacientes que utilizaram nutrição parenteral por cateter venoso central apresentaram média ( \pm desvio padrão) de idade de $54,1( \pm 17,6)$ anos, variando de 18 a 91 anos, no grupo com complicações relacionadas, e de $58,7( \pm 14,3)$ anos, com mínimo de 29 e máximo de 82 anos, no grupo sem complicações. Predominou o sexo masculino $26(72,2 \%)$ em ambos os grupos. Neoplasias gástrica $10(27,8 \%)$ e de pâncreas $5(13,9 \%)$ foram os diagnósticos mais prevalentes, com iguais frequências conforme presença de complicações relacionadas ao cateter. Destacou-se, ainda, a frequência de pacientes com Doença de Crohn que apresentaram complicações, na quantidade de 5 (13,9\%). Dentre os pacientes com complicações, 16 (44,4\%) tinham baixo peso, em comparação a $22(64,7 \%)$ no grupo sem complicações, como mostra a Tabela 1.

As principais indicações da nutrição parenteral dentre os pacientes que apresentaram alguma complicação foram fístulas no trato gastrointestinal $19(52,8 \%)$ e obstrução no trato gastrointestinal 11 (30,6\%). O número de diárias de nutrição parenteral no grupo de pacientes que tiveram complicações foi superior em 5,4 dias na média, em relação aos demais, embora não tenha sido verificada diferença significativa $(p=0,211)$. 
Foi verificada diferença estatisticamente significativa entre o número médio de sítios utilizados para inserção do cateter venoso central, sendo superior para os pacientes que apresentaram complicações $(p=0,041)$, sendo que $17(47,2 \%)$ desses participantes receberam nutrição parenteral por mais de uma via endovenosa, comparativamente a apenas 8 (23,5\%) dentre os que não tiveram complicações. Assim, utilizar dois ou mais sítios de inserção do cateter venoso central para nutrição parenteral correspondeu a uma prevalência $61,1 \%$ maior de complicações relacionadas $(R P=1,611 ; \mathrm{IC} 95 \%=1,043-2,488)$.

No que se refere aos sítios de inserção, no grupo com complicações, os mais frequentes foram subclávia direita $23(63,9 \%)$ e jugular direita $18(50,0 \%)$, predominando de forma semelhante na amostra. O acesso na jugular esquerda foi associado significativamente à ocorrência de complicações $(p=0,014)$, de modo que compreendeu uma prevalência 2 vezes maior de complicações relacionadas ( $R P=2,000 ; I C 95 \%=1,413-2,832)$ em comparação aos que não utilizaram.

A frequência de não realização de troca do cateter foi de 19 (52,8\%) dentre os que tiveram complicações e $27(79,4 \%)$ dentre os que não tiveram. Quando comparada a realização versus a não realização de troca de cateter, verificou-se uma associação estatisticamente significativa ( $p=0,024$; teste Exato de Fisher), com $\mathrm{RP}=1,715$ (IC95\%=1,116-2,635), indicando que realizar a troca correspondeu a uma prevalência $71,5 \%$ maior de complicações. Não houve associação entre a ocorrência de complicações relacionadas ao cateter venoso central utilizado para nutrição parenteral e o óbito $(p=0,845)$. Porém, destaca-se a frequência de óbitos de 22 $(61,1 \%)$ pacientes do grupo com complicações, conforme apresentado na Tabela 2.

Tabela 2 - Variáveis relacionadas à nutrição parenteral por cateter venoso central dos pacientes internados $(\mathrm{n}=70)$.

\begin{tabular}{|c|c|c|c|}
\hline Variável & $\begin{array}{c}\text { Com complicação } \\
(n=36)\end{array}$ & $\begin{array}{c}\text { Sem complicação } \\
(n=34)\end{array}$ & $p$ \\
\hline Indicação da nutrição parenteral & & & $0,409^{q}$ \\
\hline Obstrução no trato gastrointestinal & $11(30,6 \%)$ & $17(50,0 \%)$ & \\
\hline Fístulas no trato gastrointestinal I & $19(52,8 \%)$ & $11(32,4 \%)$ & \\
\hline Íleo paralítico & $1(2,8 \%)$ & $2(5,9 \%)$ & \\
\hline Síndrome do intestino curto & $2(5,6 \%)$ & $2(5,9 \%)$ & \\
\hline Desnutrição grave & $2(5,6 \%)$ & - & \\
\hline Pancreatite aguda & $1(2,8 \%)$ & $1(2,9 \%)$ & \\
\hline Síndrome disabsortiva/diarreia & & $1(2,9 \%)$ & \\
\hline Número de diárias de nutrição parenteral & $23,4 \pm 18,3$ & $18,0 \pm 17,8$ & $0,211^{t}$ \\
\hline Número de sítios de inserção utilizados & $1,6 \pm 0,6$ & $1,2 \pm 0,5$ & $0,041^{t}$ \\
\hline \multicolumn{4}{|l|}{ Sítio de inserção* } \\
\hline Jugular direita & $18(50,0 \%)$ & $20(58,8 \%)$ & $0,459^{q}$ \\
\hline Jugular esquerda & $9(25,0 \%)$ & $1(2,9 \%)$ & $0,014^{f}$ \\
\hline Subclávia direita & $23(63,9 \%)$ & $19(55,9 \%)$ & 0,4949 \\
\hline Subclávia esquerda & $6(16,7 \%)$ & $3(8,8 \%)$ & $0,479^{f}$ \\
\hline \multicolumn{4}{|l|}{ Troca de cateter } \\
\hline Uma & $14(38,9 \%)$ & $6(17,6 \%)$ & \\
\hline Duas & $3(8,3 \%)$ & $1(2,9 \%)$ & \\
\hline Não realizada & $19(52,8 \%)$ & $27(79,4 \%)$ & \\
\hline Desfecho & & & $0,845^{q}$ \\
\hline Óbito & $22(61,1 \%)$ & $20(58,8 \%)$ & \\
\hline Alta/melhora clínica/transferência & $14(38,9 \%)$ & $14(41,2 \%)$ & \\
\hline
\end{tabular}

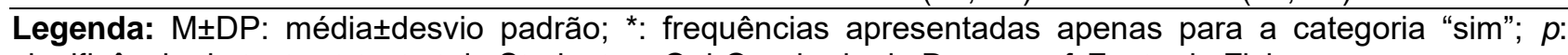
significância do teste; $t$ : teste t de Student; $q$ : Qui-Quadrado de Pearson; $f$ : Exato de Fisher.

Fonte: Alencar AS, et al., 2021.

O formulário de atividades preventivas padronizadas não foi preenchido para 60 (87.5\%) pacientes. Foram verificadas $16(44,4 \%)$ culturas de ponta de cateter e $30(83,3 \%)$ hemoculturas positivas dentre os pacientes que tiveram complicações infecciosas. Os principais gêneros de microrganismos identificados foram Staphylococcus 18 (25,7\%) e Acinetobacter 6 (8,6\%), conforme Tabela 3. 
Tabela 3 - Variáveis de investigação da complicação relacionada ao cateter venoso central dos pacientes internados $(\mathrm{n}=36)$.

\begin{tabular}{cc}
\hline Variável & $\mathbf{n}(\%)$ \\
\hline Formulário de atividades preventivas padronizadas & $10(14,3 \%)$ \\
Preenchido & $60(85,7 \%)$ \\
\hline Não preenchido & \\
\hline Ponta de cateter & $16(44,4 \%)$ \\
Positiva & $4(11,1 \%)$ \\
Negativa & $16(44,4 \%)$ \\
\hline Não realizada & \\
\hline Hemocultura & $30(83,3 \%)$ \\
Positiva & $5(13,9 \%)$ \\
Negativa & $1(2,8 \%)$ \\
Não realizada & \\
\hline Gênero do microrganismo* & $18(25,7 \%)$ \\
Staphylococcus & $6(8,6 \%)$ \\
Acinetobacter & $3(4,3 \%)$ \\
Candida & $2(2,9 \%)$ \\
Enterobacter & $5(7,1 \%)$ \\
Klebsiella & $2(2,9 \%)$ \\
Pseudomonas & $2(2,9 \%)$ \\
Serratia & $1(1,4 \%)$ \\
Kodamaea & $1(1,4 \%)$ \\
Morganella &
\end{tabular}

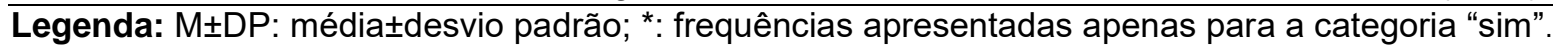

Fonte: Alencar AS, et al., 2021.

As espécies mais frequentes foram Staphylococcus epidermidis 11 (15,7\%), seguido de Acinetobacter baumannii 6 (8,6\%), Klebsiella pneumoniae 5 (7,1\%) e Staphylococcus aereus 4 (5,7\%). Destacaram-se, ainda, a quantidade de Staphylococcus sp. identificadas, como apresentado na Figura 1.

Figura 1 - Espécies de microrganismos causadores das infecções relacionadas ao cateter venoso central dos pacientes internados $(n=36)$.

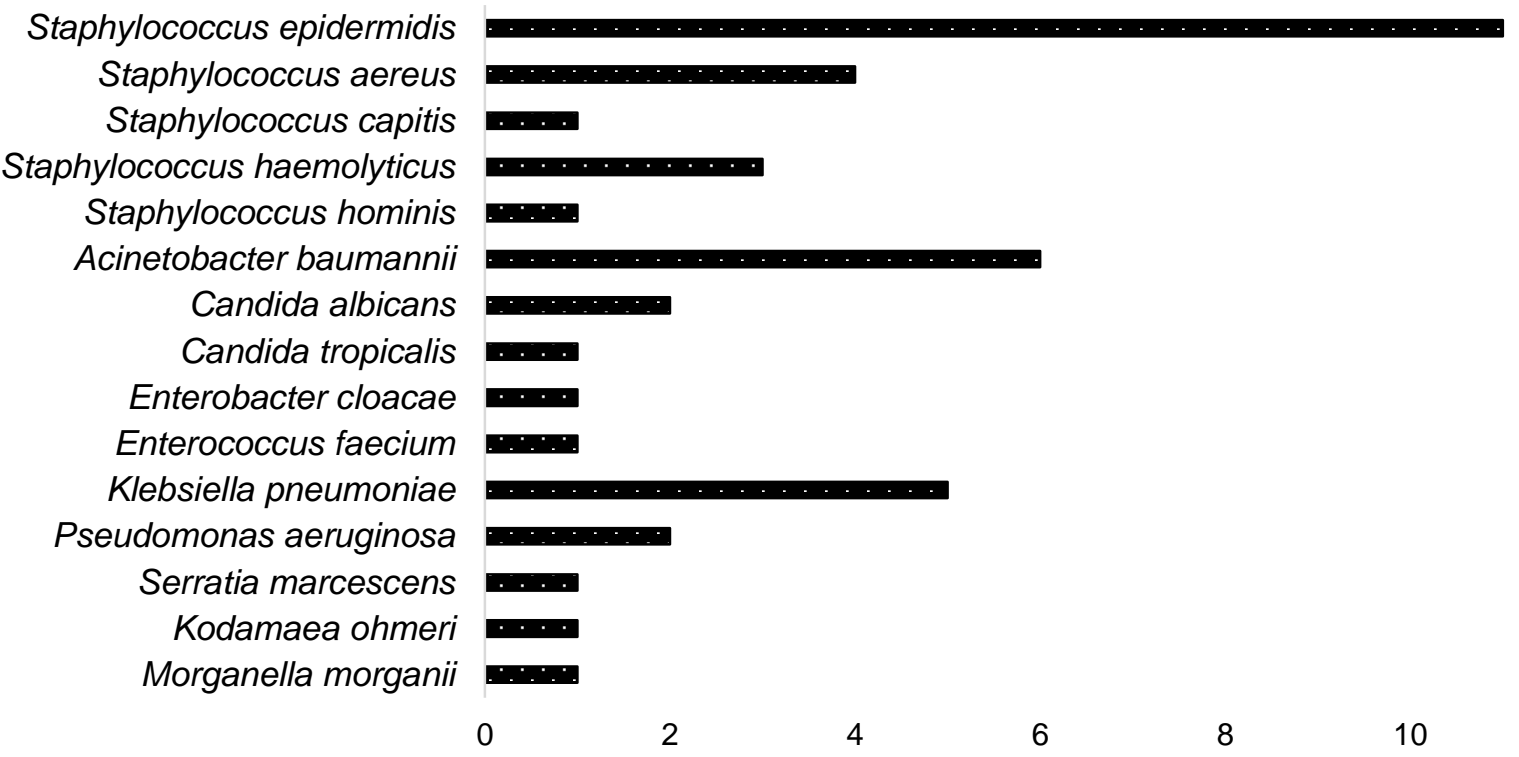

Fonte: Alencar AS, et al., 2021. 


\section{DISCUSSÃO}

Os resultados apontaram que complicações relacionadas ao cateter venoso central utilizado para nutrição parenteral ocorreram em $51,4 \%$ dos pacientes internados no período avaliado, em que todas foram infecciosas. Em um dos casos, a complicação infecciosa ocorreu concomitantemente a uma intolerância hepática. A literatura confirma a nutrição parenteral como um fator de risco significativo para infecção de cateter, pois apresenta aminoácidos e dextrose, que favorecem o crescimento de bácterias e fungos (COMERLATO PH, et al., 2020; FONSECA G, et al., 2018; OPILLA M, et al., 2008).

Além disso, pacientes usuários de nutrição parenteral geralmente possuem quadro clínico grave, internação prolongada, são desnutridos e imunodeprimidos, o que favorece a ocorrência de complicações infecciosas. Outro fator de risco importante é que o estado crítico do paciente favorece a hiperglicemia que, juntamente com a administração de dextrose, favorece o crescimento bacteriano. A infecção também pode ocorrer por bactérias da pele, por quebra da técnica de inserção do cateter e na manipulação do mesmo (OPILLA M, et al., 2008).

No México, a taxa de infecções da corrente sanguínea relacionadas ao cateter utilizado para nutrição parenteral foi de 19\%, em que os microrganismos mais frequentes foram Staphylococcus sp. (44\%) e Candida sp. (25\%) (PARRA-FLORES M, et al., 2017). Nos Estados Unidos, entre 38.674 pacientes com cateteres centrais, 9,1\% desenvolveram infecções da corrente sanguínea, sendo que $21,8 \%$ destas foram relacionadas ao cateter utilizado para nutrição parenteral, a qual foi um fator de risco independente para o desenvolvimento de complicações infecciosas (FONSECA G, et al., 2018).

No Brasil, uma coorte retrospectiva realizada no Rio Grande do Sul, analisou 165 pacientes internados em um hospital universitário, com um total de 247 cateteres utilizados para nutrição parenteral, e encontrou uma prevalência de infecção da corrente sanguínea associada ao cateter de 14,5\% (COMERLATO PH, et al., 2020). Em Brasília, estudo realizado em unidades de terapia intensiva de um hospital geral público verificou uma prevalência de 6,6\% (SILVA RF, et al., 2020). A prevalência muito superior encontrada no presente estudo pode ter decorrido das características gerais de gravidade dos pacientes, assim como alertam para a necessidade de revisão e seguimento dos protocolos de prevenção.

No presente estudo, os principais diagnósticos dos pacientes internados e que necessitaram de nutrição parenteral foram neoplasias gástrica e de pâncreas, além de ter sido verificado um importante percentual de pacientes com baixo peso $(44,4 \%)$, porém, sem associação com a presença de complicações relacionadas ao cateter venoso central. O câncer é uma das principais causas de morte no mundo, de causa multifatorial que envolve genética, fatores modificáveis de hábitos, e meio ambiente. Em pacientes oncológicos, os distúrbios nutricionais são frequentes e podem decorrer da baixa ingestão ou absorção de nutrientes, causando distúrbios metabólicos e alteração do funcionamento celular (SILVA IBP, et al., 2020). Ademais, múltiplos fatores favorecem o quadro infecioso, como tempo de internação prolongado, tratamento agressivo, uso de antimicrobianos, necessidade de hemotransfusão e imunossupressão (ROLSTON KV, et al., 2017).

O trato gastrointestinal é a primeira escolha para garantir o aporte nutricional. Em caso de impossibilidade da via oral e enteral, é realizada a administração parenteral de nutrientes para prevenir a deteriorização do estado nutricional e minimizar as complicações do jejum prolongado. As principais indicações da nutrição parenteral foram fístulas e obstrução do trato gastrointestinal. Fístula é um canal patológico que cria uma comunicação entre duas vísceras ou entre uma víscera e a pele, e está relacionada à desnutrição, distúrbios hidroeletrolíticos e sepse e, com isso, apresenta alto índice de mortalidade (ITZHAKI M, et al., 2020).

No presente estudo, os sitíos de inserção preferenciais foram as veias subclávia e jugular direitas. Contudo, houve uma frequência significativa de complicações relacionadas ao cateter venoso central inserido na veia jugular esquerda. A literatura aponta que, na maioria dos casos, a via central é executada por meio de canulação da via subclávica através da via infraclavicular e rotineiramente colocada em condições de acesso à veia cava superior. A subclávia é preferida por proporcionar melhor conforto ao paciente, maior facilidade de manuseio para curativo e fixação, além de melhor identificação anatômica em pacientes obesos. O lado a ser puncionado deve ser o direito por diminuir o risco de pneumotórax, pois a cúpula pleural é mais baixa e há menor possibilidade de mau posicionamento, devido o trajeto até o átrio direito ser mais retilíneo (SILVA IBP, et al., 2020). 
Houve, ainda, diferença estatisticamente significativa entre o número de sítios de inserção utilizados e a ocorrência de complicações, sendo superior no grupo de pacientes que adquiriram infecção. Esses dados podem estar relacionados à maior manipulação dos cateteres, maior tempo de internação hospitalar e maior tempo de utilização de nutrição parenteral. Estudo prospectivo realizado em Sergipe que avaliou os cuidados com cateter em unidades de terapia intensiva evidenciou uma baixa adesão na lavagem das mãos e na desinfecção de materiais durante a troca de curativo e de equipos. Esses resultados são preocupantes, pois a higienização das mãos é a medida mais importante no controle de infecções nos serviços de saúde (LLAPARODRIGUES EO, et al., 2020).

Embora não tenha sido verificada associação entre a ocorrência de complicações relacionadas ao cateter venoso central utilizado para nutrição parenteral, destaca-se a mortalidade de $61,1 \%$ dos pacientes do grupo com complicações e $58,8 \%$ dos pacientes que não apresentaram complicações infecciosas. Estudos confirmam a associação entre a ocorrência de complicações relacionadas ao cateter venoso central utilizado para nutrição parenteral e uma maior taxa de mortalidade (BOLL B, et al., 2021; TATSUNO K, et al., 2019). Um estudo realizado em um hospital de urgência de Teresina evidenciou o desfecho óbito em $51,14 \%$ dos pacientes avaliados e apontou que a mortalidade pode estar relacionada à gravidade da patologia de base do paciente ou às complicações do uso da nutrição parenteral (CARVALHO LHS, et al., 2020).

Estudo brasileiro verificou associações entre a ocorrência de infecção da corrente sanguínea associada ao cateter central para uso de nutrição parenteral e um maior tempo de internação, maior tempo de nutrição parenteral, maior tempo de cateter, cuja mortalidade geral foi de $24,8 \%$ e apenas o índice de comorbidade dos pacientes foi associado à morte (COMERLATO PH, et al., 2020). A alta mortalidade encontrada entre os pacientes internados no hospital em foco pode ser um indicativo da gravidade da condição clínica dos mesmos, especialmente ao considerar a elevada frequência de neoplasias e doenças graves do trato gastrointestinal.

Foi constatado que $85,7 \%$ dos formulários preventivos de inserção de cateter central não foram preenchidos, o que pode indicar possíveis falhas nas medidas preventivas das infecções de corrente sanguínea, destacando-se como elementos fundamentais na inserção do cateter a retirada de adornos, higienização das mãos, uso de clorexidina para preparo da pele, uso de barreira máxima de precaução, utilização de campo estéril que cubra todo o corpo do paciente e realização de curativo oclusivo com gaze e fita adesiva estéril. Diante disso, reforça-se a recomendação da literatura quanto ao desenvolvimento de estratégias de educação continuada para garantir o preenchimento do checklist, a fim de proporcionar maior segurança aos pacientes (AL-TAWIL ES, et al., 2016). A prevenção inclui medidas de intervenção e vigilância em todas as etapas do procedimento, incluindo a etapa da inserção do cateter central (OPILLA M, et al., 2008).

Os microrganismos mais comuns nas infecções relacionadas ao cateter foram Staphylococcus epidermidis (15,7\%), seguido de Acinetobacter baumannii $(8,6 \%)$, Klebsiella pneumoniae $(7,1 \%)$ e Staphylococcus aereus (5,7\%). Evidenciou-se, ainda, a alta frequência de Staphylococcus sp. Os resultados encontrados corroboraram com estudo realizado no México, em que o Staphylococcus epidermidis foi apontado como o principal agente relacionado às infecções em pacientes de baixo peso e usuários de formulações parenterais (PARRA-FLORES M, et al., 2017).

A preparação dos profissionais de enfermagem que manipulam cotidianamente esses cateteres e a existência de pragmáticas rigorosas de cuidados são essenciais, pois são contrabalanceadas pelo risco adicional de complicações infecciosas. O procedimento estéril e a antissepsia da pele são considerados intervenções importantes na prevenção de infecção (LLAPA-RODRIGUES EO, et al., 2019; SILVESTRE OP, et al., 2018).

Diante dos dados deste estudo, salientamos a orientação dos pacientes para cuidar melhor da sua higiene, principalmente das mãos. Apesar dos benefícios da utilização dos cateteres vasculares, não há como descartar os riscos. Ademais, visto que o protocolo de cuidados de cateteres venosos centrais permanentes é primordial para a prevenção de infecção e para a extensão do tempo de uso desses cateteres, permitindo a continuidade da oferta de nutrientes fundamentais à vida (BYERS K, et al., 1995), é imperativa a revisão de protocolos preexistentes, bem como a fiscalização de sua implementação pelos profissionais envolvidos no cuidado direto aos pacientes. 


\section{CONCLUSÃO}

A prevalência de complicações dos cateteres de acesso venoso central para uso de nutrição parenteral foi alta e do tipo infecciosa. Um maior número de sítios de inserção, acesso em jugular esquerda e realização de troca de cateter foram relacionados à ocorrência de complicações. Os microorganismos isolados mais frequentes foram do gênero Sthaphylococus, com predominância do $S$. epidermidis. Os resultados obtidos denotaram a importância da nutrição parenteral para garantir o estado nutricional de pacientes bastante debilitados. Porém, evidenciaram a urgente necessidade de revisão dos protocolos institucionais, tendo em vista que a prevalência dessas complicações pode ser reduzida quando a equipe médica realiza a inserção do cateter seguindo as normas de vigilância e os cuidados contínuos são realizados para garantir a segurança do paciente.

\section{REFERÊNCIAS}

1- AKARABORWORN O. A review in emergency central venous catheterization. Chin J Traumatol. 2017; 20(3): 137-40.

2- AL-TAWIL ES, et al. Catheter-related blood stream infection in patients receiving long-term home parenteral nutrition: Tertiary care hospital experience in Saudi Arabia. Saudi J Gastroenterol. 2016; 22(4): 304-8.

3- BYERS K. Case fatality rate for catheter-related bloodstream infections: A meta-analysis. Proceedings of the 5th annual meeting of the Society for Hospital Epidemiology of America. San Diego, CA, 1995.

4- BÖLL B, et al. Central venous catheter-related infections in hematology and oncology: 2020 updated guidelines on diagnosis, management, and prevention by the Infectious Diseases Working Party (AGIHO) of the German Society of Hematology and Medical Oncology (DGHO). Ann Hematol. 2021; 100(1): 239-59.

5- CARVALHO LHS, et al. Perfil dos usuários de terapia nutricional parenteral em um hospital de Teresina-PI. Rev Int Ciênc Méd. 2020; 2020: 1-5.

6- COMERLATO PH, et al. Complications of central venous catheter insertion in a teaching hospital. Rev Assoc Med Bras. 2017; 63(7): 613-20.

7- COMERLATO PH, et al. Infectious complications associated with parenteral nutrition in intensive care unit and nonintensive care unit patients. Braz J Infect Dis. 2020; 24(2): 137-43.

8- EASTRIDGE BJ, LEFOR AT. Complications of indwelling venous access devices in cancer patients. J Clin Oncol. 1995; 13(1): 233-8.

9- FONSECA G, et al. The Relationship Between Parenteral Nutrition and Central Line-Associated Bloodstream Infections: 2009-2014. JPEN J Parenter Enteral Nutr. 2018; 42(1): 171-5.

10-ITZHAKI M, SINGER P. Advances in Medical Nutrition Therapy: Parenteral Nutrition. Nutrients. 2020; $12(3)$ : e717.

11-LAMBELL KJ, et al. Nutrition therapy in critical illness: a review of the literature for clinicians. Crit Care. 2020; 24(1): 35-42.

12-LLAPA-RODRÍGUEZ EO, et al. Safety in equipment and dressing changes for central vascular catheters: an observational study. Text Cont Enf. 2020; 29: 1-15.

13-MIYAO, H. Safety committee of Japanese society of anesthesiologists blood transfusion, colloid therapy and the possible savina of albumin volumes durina suraerv: data analysis of the survey for certified hospitals of the Japanese society of anesthesiologists. J Anesth. 2016; 30: 384-390.

14-OPILLA M. Epidemiology of bloodstream infection associated with parenteral nutrition. Am J Infect Control. 2008; 36(10): 5-8.

15-PATEL AR, et al. Central Line Catheters and Associated Complications: A Review. Cureus. 2019; 11 (5): e4717.

16-PARRA-FLORES M, et al. Incidence of catheter-related infection incidence and risk factors in patients on total parenteral nutrition in a third level hospital. Cir Cir. 2017; 85(2): 104-8.

17-PIRONI L, et al. ESPEN guideline on home parenteral nutrition. Clin Nutr. 2020; 39(6): 1645-66.

18-ROLSTON KV. Infections in Cancer Patients with Solid Tumors: A Review. Infect Dis Ther. 2017; 6(1): 69-83.

19-SILVA IBD, et al. Evaluation of the nutritional profile of oncological patients in two hospital institutions: case series. Braz J Develop. 2020; 6(9): 68447-59.

20-SILVA RF, et al. Clinical conditions and complications associated with parenteral nutrition use in critically ill patients admitted to an intensive care unit of a general hospital. Nutr Hosp. 2020; 37(4): 645-53.

21-SILVA J, MIRANDA SR, SOUSA CY. Preoperative nutritional risk through total lymphocyte content, serum albumine levels and strongkids tribal tool in children submitted to cardiac surgeries. Nutr clín diet hosp. 2019; 39(3): 50-7.

22-SILVESTRE OP, et al. Microorganismos isolados de pontas de cateteres venosos central e perfil de suscetibilidade antimicrobiana. Rev Saúde Com UNESPAR. 2018; 1(1): 3-11.

23-SOU V, et al. A clinical pathway for the management of difficult venous access. BMC Nurs. 2017; 16: 64-9.

24-TATSUNO K, et al. Clinical Features of Bloodstream Infections Associated with Peripheral Versus Central Venous Catheters. Infect Dis Ther. 2019; 8(3): 343-52.

25-VASHI PG, et al. Incidence of and factors associated with catheter-related bloodstream infection in patients with advanced solid tumors on home parenteral nutrition managed using a standardized catheter care protocol. BMC Infect Dis. 2017; 17(1): 372-8.

26-WEBER TK, et al. Nutritional therapy in hospital care of in-patients with Covid-19: evidence, consensus and practice guidelines. Rev Nutr. 2020; 33: e200212.

27- WEIR CB, JAN A. BMI Classification Percentile And Cut Off Points. In: StatPearls. Treasure Island (FL): StatPearls Publishing; 2020.

28-ZERATI AE, et al. Totally implantable venous catheters: history, implantation technique and complications. J vasc bras. 2017; 16(2): 128-3. 